\title{
Arbeitszeitsituation und betriebliche Interessenvertretung - Bessere Zeiten mit Betriebsrat?
}

\author{
Peter Ellguth \\ Markus Promberger
}

\begin{abstract}
Die Ausgestaltung des betrieblichen Arbeitszeit-Reglements ist ein zentrales Thema in der anhaltenden Flexibilisierungsdebatte und nicht nur für die Betriebe, sondern auch für die Beschäftigten von großer Bedeutung. Dank entsprechender Mitbestimmungsrechte kann der Betriebsrat darauf wesentlichen Einfluss nehmen - so er denn existiert. Gibt es Unterschiede in der Arbeitszeitsituation von Betrieben mit und ohne Betriebsrat? Welche Motive und Wirkungsweisen lassen sich dabei identifizieren und was bedeutet dies für die Beschäftigten?
\end{abstract}

\section{Einleitung}

Seit fast 25 Jahren sind die betrieblichen Arbeitszeiten immer wieder Gegenstand von Debatten in Wissenschaft, Wirtschaft und Politik. Gewerkschaftlichen Verkürzungsforderungen und Bestrebungen nach selbstbestimmter Zeitnutzung der Beschäftigten stehen unternehmerische Flexibilisierungsforderungen, seit einigen Jahren auch politische Interessen an längeren Arbeitszeiten und damit indirekten Lohnsenkungen gegenüber. Dabei lassen sich phasenweise unterschiedliche Betonungen der einzelnen Motive - neben teilweise überraschenden Parallelen zu Arbeitszeitkonflikten früherer Epochen - feststellen (Promberger et al. 2002; Promberger 2004). Historisch neu ist beim Arbeitszeitkonflikt von 1984 bis heute die starke Rolle der betrieblichen Regelungsebene (vgl. zur Verbetrieblichung: Schmidt/Trinczek 1988).

Die Forschung beobachtet dieses Terrain seit Langem, und zwar in zwei Strängen, dem älteren der empirischen Mitbestimmungsforschung, dessen Wurzeln bereits in den 1920er Jahren liegen, und der Arbeitszeitforschung, die erst in den 1980er Jahren als eigenständiges Feld Profil gewann, jedoch gewissermaßen von Geburt an starke Berührungen mit dem Themengebiet der „industriellen Beziehungen“ aufwies. Die Geschichte der deutschen Mitbestimmungsforschung war lange Jahre geprägt von einem fruchtbaren Nebeneinander aus idealtypischen Überlegungen
(Neuloh 1956; Fürstenberg 1958), Fallstudienuntersuchungen unterschiedlichster Zusammensetzung (Pirker et al. 1955; Popitz et al. 1964; Bergmann et al. 1975; Kotthoff 1981; Bosch et al. 1999) und vergleichsweise simplen standardisierten Befragungen (Mausolff 1952; Blume 1964). Ähnliches gilt für die Arbeitszeitforschung, in der bis in die neueste Zeit ebenfalls Fallstudien (Lehndorff 1997; Lindecke 2000; Promberger et al. 2002; vgl. auch den Überblick bei Frege 2002) neben einfach aufbereiteten standardisierten Umfragedaten (Bosch et al. 1988; Promberger 1993; Herrmann et al. 1999) stehen. Der Entwicklung der „labour economics“ als wissenschaftliche Disziplin, aber nicht zuletzt auch der mittlerweile gestiegenen Rechnerleistung verdanken wir in neuester Zeit den jüngsten, dritten Typus von Forschung zu Arbeitszeit und/oder Mitbestimmung: die ökonometrische bzw. multivariat-statistische Analyse großer Datensätze aus Befragungen und Verwaltungsregistern (vgl. für einen Überblick: Hübler 2003; Jirjahn 2005). Eigentümlich für die gegenwärtige Forschungslandschaft zum Themenkomplex Arbeitszeit und Mitbestimmung im Betrieb scheint uns dabei zu sein, dass Verbindungen zwischen den ersten beiden und dem dritten Typus von Forschung bislang Seltenheitswert besitzen - auch wenn die wechselseitige Rezeption allmählich wächst (Jirjahn 2005). Auch hierzu will dieser Aufsatz beitragen.

Ausgehend von den Erkenntnisbeständen der industriesoziologischen und arbeitsökonomischen Betriebsrats- und Arbeitszeitforschung sollen deshalb zunächst Vorüberlegungen über mögliche Wechsel- beziehungen zwischen der Existenz von Betriebsräten und der Ausformung der betrieblichen Arbeitszeitsituation entwickelt werden (Abschnitt 2), die dann auf der Grundlage des IAB-Betriebspanels zu überprüfen sind. Anschließend daran werden die methodischen Überlegungen vorgestellt, die den verwendeten Matchingverfahren zugrunde liegen. Sie erlauben es, durch die Gegenüberstellung von Betriebsratsbetrieben mit ihren statistischen Zwillingen gleicher Größe, Branche, Belegschaftsstruktur etc., jedoch ohne Betriebsrat, bestimmte betriebliche Arbeitszeitmuster mit der Existenz von Betriebsräten in Beziehung zu setzen (Abschnitt 3). Abschließend werden die Ergebnisse dieser Matched-Pair-Analysen präsentiert (Abschnitt 4).

Peter Ellguth, Wissenschaftlicher Mitarbeiter
im Forschungsbereich „Betriebe und
Beschäftigung“, Institut für Arbeitsmarkt-
und Berufsforschung (IAB) der Bundes-
agentur für Arbeit.
Arbeitsschwerpunkte: Arbeitsbeziehungen
und betriebliche Arbeitszeitpolitik.
e-mail: peter.ellguth@iab.de
Markus Promberger, Dr., Leiter des
Forschungsbereichs „Erwerbslosigkeit und
Teilhabe“, Institut für Arbeitsmarkt- und
Berufsforschung (IAB) der Bundesagentur
für Arbeit. Arbeitsschwerpunkte: Arbeitszeit,
Mitbestimmung, atypische Beschäftigung,
Erwerbslosenforschung, Arbeitsleben im
Wandel, Sozialgeschichte der Arbeit.
e-mail: markus.promberger@iab.de

Peter Ellguth, Wissenschaftlicher Mitarbeiter im Forschungsbereich "Betriebe und Beschäftigung ", Institut für Arbeitsmarkt und Berufsforschung (IAB) der Bundes agentur für Arbeit. Arbeitsschwerpunkte: Arbeitsbeziehungen und betriebliche Arbeitszeitpolitik. e-mail: peter.ellguth@iab.de Markus Promberger, Dr., Leiter des Erwerbslosigkeit und Berufsforschung (IAB) der Bundesagentur für Arbeit. Arbeitsschwerpunkte: Arbeitszeit, Mitbestimmung, atypische Beschäftigung, Wandel, Sozialgeschichte der Arbeit. e-mail: markus.promberger@iab.de 


\section{Arbeitszeiten und Betriebs- räte - eine empirisch offene Frage}

Das Betriebsverfassungsgesetz (BetrVG), das zuletzt im Juli 2001 reformiert wurde, beschreibt zum einen den institutionellen Rahmen für die Einrichtung und Ausgestaltung von Betriebsratsgremien, zum anderen bildet es die rechtliche Grundlage für das betriebspolitische Handeln des Betriebsrats. Vor allem in Bezug auf personelle Einzelmaßnahmen ( $\$ 99$ und $\$ 102$ BetrVG) und insbesondere Veränderungen der Arbeitszeiten ( $\$ 87$ BetrVG) kann er entsprechende Vorhaben wenn nicht verhindern, so doch verzögern und verteuern.

Der potenzielle Einfluss des Betriebsrats beschränkt sich aber nicht auf diese eng umrissenen Inhalte. Durch die Kopplung der Zustimmung zu bestimmten mitbestimmungspflichtigen Maßnahmen der Geschäftsleitung an Zugeständnisse auf anderen, dem unmittelbaren Machtbereich des Betriebsrats entzogenen Regelungsgebieten - sogenannte „package-deals“ (Müller-Jentsch 1995, S. 66) - kann er seinen faktischen Einfluss auch auf andere Handlungsfelder ausdehnen. Nicht zuletzt sind Betriebsräte - wie bereits Kotthoff (1979) feststellte - trotz formaler Trennung im dualen System (zum Begriff des dualen Systems vgl. Bergmann et al. 1975; Schmidt/ Trinczek 1991) der ,industrial relations“ in Deutschland faktisch das Rückgrat der gewerkschaftlichen Organisation im Betrieb. Überdies sollen Betriebsräte auch mittels Betriebsverfassungsgesetz die Einhaltung gesetzlicher und tariflicher Normen überwachen.

Erschwerend kommt jedoch hinzu, dass die Möglichkeiten des Betriebsrats zur Einflussnahme, sei es auf Basis gesetzlich definierter Rechte oder mittels „Koppelgeschäften", noch nichts über seine faktische Wirkung aussagt. Wie die Ergebnisse qualitativer Betriebsratsforschung zeigen, ist die tatsächliche betriebspolitische Bedeutung des Betriebsrats von der Ausgestaltung der innerbetrieblichen Austauschbeziehungen, das heißt den spezifischen Handlungsstrukturen und Beziehungen zwischen Interessenvertretung und Management, abhängig und damit ex ante nicht bestimmbar (Kotthoff 1981, 1994; Bosch et al. 1999; Müller-Jentsch/Seitz 1998);
Fallstudien zur betrieblichen Arbeitszeitpolitik bestätigen dies (Promberger et al. 2002; Lindecke 2000). Auch die Arbeitsökonomik geht von einer Vielzahl möglicher Funktionen und Effekte des Betriebsrats aus, deren Bandbreite zwar theoretisch bestimmbar ist, deren tatsächliche Ausprägungen jedoch nur empirisch geklärt werden können (Jirjahn 2005). Die naive Annahme, man müsse die Wirkung von Betriebsräten nicht untersuchen, denn die Antwort der Theorie sei eindeutig, hat ihre Gültigkeit spätestens seit Freeman/Lazear (1995) verloren.

Vor diesem Hintergrund stellen wir die Frage, ob die Existenz eines Betriebsrats die betriebliche Arbeitszeitsituation verbessert, im Sinne eines häufigeren Vorhandenseins von formalen Regelungen arbeitszeitpolitischer Tatbestände oder eines selteneren Auftretens gemeinhin als problematisch empfundener und beschriebener Arbeitszeitformen, bei ansonsten weitgehend gleichen Bedingungen.

Nach den Ergebnissen des IAB-Betriebspanels 2005 existiert nur in ca. jedem neunten betriebsratsfähigen Betrieb ( $a b$ fünf Beschäftigte) der Privatwirtschaft tatsächlich auch ein Betriebsrat. In diesen Betrieben arbeiten allerdings rund $46 \%$ der Beschäftigten. Die starke Größenabhängigkeit, die deutlichen branchenspezifischen Unterschiede und die nach wie vor bestehenden West-Ost-Differenzen in der Verbreitung von Betriebsratsgremien sind bereits hinlänglich dokumentiert und brauchen an dieser Stelle nicht wiederholt werden (vgl. ausführlich: Ellguth 2007).

Die Datenlage hinsichtlich betrieblicher Arbeitszeiten in Deutschland ist vergleichsweise überschaubar (Ellguth/Promberger 2004a). Vor allem hinsichtlich der Samplegröße, der längsschnittlichen Auswertbarkeit und der Einbeziehung von Betrieben mit und ohne Betriebsrat bietet sich nur das IAB-Betriebspanel an: Es umfasst in repräsentativer Weise alle Betriebe mit sozialversicherungspflichtiger Beschäftigung - mit Ausnahme des Bergbaus und der privaten Haushalte, wo nur eingeschränkte Repräsentativität vorliegt.

Welche betrieblichen Arbeitszeitvariablen bieten sich für eine Analyse im IABBetriebspanel 2006 an? Dies sind zunächst die vereinbarte Wochenarbeitszeit für Vollzeitbeschäftigte, dann das Vorhandensein von Arbeitszeitkonten und deren Ausmaß, der Einsatz von Teilzeitbeschäftigung, sei es als sozialversicherungspflichtige oder ge- ringfügige Beschäftigung, sowie eine - notwendigerweise etwas unzulängliche - Abfrage der Überstundensituation. Unzulänglich deshalb, weil aus methodischen Gründen auf eine Volumenabfrage verzichtet wurde. Darüber hinaus liegen unter anderem Informationen zur Nutzung von Vertrauensarbeitszeit, Samstags- und Sonntagsarbeit, sowie zum Einsatz beschäftigungssichernder Arbeitszeitverkürzung vor.

Nach unserer Rechnung beträgt die regelmäßige, vertraglich vereinbarte Wochenarbeitszeit von Vollzeitbeschäftigten in den privatwirtschaftlichen Betrieben Westdeutschlands im Jahre 2006 39,2 Stunden, in Ostdeutschland hingegen 39,8 Stunden. ${ }^{1}$ Andere Erhebungen weisen aus methodischen und technischen Gründen deutlich niedrigere Werte als das IAB-Betriebspanel aus (Ellguth/Promberger 2004a).

Arbeitszeitkonten sind aus der betrieblichen Praxis nicht mehr wegzudenken. So praktizieren rund $21 \%$ aller privatwirtschaftlichen Betriebe im Osten und rund $20 \%$ im Westen Arbeitszeitkonten. Abweichende Zahlen in anderen Befragungen ergeben sich durch die stärkere Berücksichtigung von Klein- und Kleinstbetrieben im IAB-Betriebspanel. Betrachtet man nur betriebsratsfähige Betriebe, so erhöhen sich die Anteilswerte auf 32 \% bzw. 27 \%. Bekanntermaßen sind Arbeitszeitkonten vor allem in Mittel- und Großbetrieben üblich, während in Kleinbetrieben andere, vor allem informelle Arten der Arbeitszeitregulierung dominieren.

Interessant ist neben der Verbreitung der Arbeitszeitkonten auch die Nutzungsintensität in den Betrieben - man kann dazu auch „Inklusionsgrad“ sagen. Hier geht es darum, welcher Anteil der Belegschaft in die Nutzung der Konten einbezogen ist. Der Inklusionsgrad liegt durchschnittlich bei etwa $85 \%$ der Belegschaft, wobei aus der vorliegenden Fallstudienevidenz (Promberger et al. 2002; Lindecke 2000; Herrmann et al. 1999) deutlich wird, dass eine Einbeziehung eines bestimmten Teils der Belegschaft nicht notwendig bedeutet, dass ein und dieselbe Kontenregelung für all diese Mitarbeiter gilt.

\footnotetext{
Es handelt sich dabei um aggregierte Angaben zu den betriebsdurchschnittlichen, vereinbarten Arbeitszeiten auf Basis der Arbeitgeberangaben im IAB-Betriebspanel, nicht um tatsächlich geleistete Arbeitszeiten oder tarifliche Arbeitszeiten.
} 
Teilzeitarbeit ist weitverbreitet: Über die Hälfte aller Betriebe der Privatwirtschaft in Ostdeutschland (55\%) und drei Viertel $(75 \%)$ in Westdeutschland praktizieren diese Arbeitszeitform. Unter dem Etikett „Teilzeitarbeit“ firmieren jedoch höchst unterschiedliche Tatbestände, was Arbeitszeitlänge, soziale Absicherung und betriebliche Sozialintegration betrifft (Ellguth/Promberger 2004a).

Rund $44 \%$ aller privatwirtschaftlichen Betriebe praktizieren Überstunden. Erwartungsgemäß finden wir eine stark zunehmende Verbreitung dieses Instruments mit wachsender Betriebsgröße, aber auch eine starke Branchenstreuung, die sich nur teilweise in der Betriebsgröße auflöst. Durchschnittlich leisten $61 \%$ aller Mitarbeiter in ostdeutschen Überstundenbetrieben bezahlte Überstunden, im Westen sind es nur rund $55 \%$. In jedem vierten ostdeutschen und jedem sechsten westdeutschen Überstundenbetrieb (mit bezahlten Überstunden) leisten alle Beschäftigten bezahlte Überstunden.

Vertrauensarbeitszeit wird von rund $15 \%$ aller Betriebe der Privatwirtschaft praktiziert, in Westdeutschland und in größeren Betrieben trifft man diese Arbeitszeitform überdurchschnittlich häufig an. Der Inklusionsgrad nimmt hingegen mit steigender Betriebsgröße und der zunehmenden funktionalen und sozialen Differenzierung ab.

Jeder dritte deutsche privatwirtschaftliche Betrieb praktiziert Samstagsarbeit, jeder achte Sonntagsarbeit - wobei nicht nach der Häufigkeit, sondern nur nach dem prinzipiellen Vorhandensein gefragt wurde. Deutliche Unterschiede zwischen Ost und Westdeutschland bestehen hierbei nicht. Die Zusammenhänge mit der Betriebsgröße sind uneinheitlich.

In der Fläche sind beschäftigungssichernde Arbeitszeitverkürzungen nur gering verbreitet: Rund $2 \%$ aller Betriebe reduzieren die Arbeitszeiten ihrer Belegschaften im „Tausch“ gegen Arbeitsplatzgarantien. Größere Betriebe nutzen dieses Instrument jedoch wesentlich häufiger, sodass weit mehr Beschäftigte in solche Regelungen einbezogen sind, als die geringen Anteilswerte in der Fläche nahelegen.

Wie die Verbreitung und Nutzung dieser Arbeitszeitformen mit der Existenz von Betriebsräten zusammenhängen, wird im Folgenden analysiert.

\section{Untersuchungsfeld und methodischer Zugang}

Unser Untersuchungsfeld sind die privatwirtschaftlichen Betriebe in den alten und neuen Bundesländern. Die Auswertungen zu den Wirkungen eines Betriebsrats werden nur für die Betriebe ab fünf Beschäftigten durchgeführt. Die Datenbasis bildet die Welle 2006 des IAB-Betriebspanels, ${ }^{2}$ die sich durch einen Befragungsschwerpunkt zu betrieblichen Arbeitszeiten auszeichnet. Die Analysen werden getrennt für West- und Ostdeutschland durchgeführt, weil zum einen nach wie vor Unterschiede in der Mitbestimmungspraxis bestehen (Bosch et al. 1999; Artus et al. 2001; Artus 2003); und sich zum anderen auch - wie wir gesehen haben - zum Teil deutliche Differenzen in der betrieblichen Arbeitszeitsituation in beiden Landesteilen zeigen (Ellguth/Promberger 2004a).

Gegenstand unserer Untersuchung ist der mögliche Einfluss (der Existenz) eines Betriebsrats auf die betriebliche Arbeitszeitpolitik. Etwas zurückhaltender formuliert interessiert uns die Frage, wie sich Betriebe mit Betriebsrat von solchen ohne in ihrem Arbeitszeitregime unterscheiden. Abweichend von den sonst für solche Fragestellungen üblichen ökonometrischen Verfahren (vor allem probit- und tobit-Modelle), bei denen der Betriebsrat als unabhängige (dummy-) Variable neben anderen in die Schätzung der Bestimmungsgründe eines bestimmten Merkmals, z. B. der Existenz von Arbeitszeitkonten, eingeht, soll hier ein alternativer methodischer Zugang verfolgt werden. Dazu wird auf die bislang vor allem aus der Evaluationsforschung bekannten Matching-Verfahren (Methoden der Paarbildung) zurückgegriffen. ${ }^{3}$

Die Grundidee hinter den MatchingVerfahren ist die Bildung einer Kontrollbzw. Vergleichsgruppe zu derjenigen mit dem interessierenden Merkmal (in unserem Fall Betriebe mit Betriebsrat) aus dem Reservoire der Betriebe, die diese Eigenschaft nicht aufweisen (Betriebe ohne Betriebsrat). Dabei sollen die ausgewählten Einheiten eine möglichst große Ähnlichkeit mit den Betriebsratsbetrieben aufweisen. Nach erfolgreicher Paarbildung ist ein Vergleich der beiden (nun weitgehend identischen) Gruppen von Betrieben entlang interessierender „Performance-Varia- blen“ möglich. Das Problem, dass sich Folgerungen über den Einfluss eines Betriebsrats aus einer "naiven“ Gegenüberstellung aller Betriebe mit und ohne Betriebsrat verbieten, da beobachtbare Unterschiede in interessierenden Variablen vermutlich auch auf andere Faktoren zurückzuführen sind, ist damit obsolet.

Für die Durchführung des Matching existieren eine Reihe alternativer Verfahren, deren Diskussion im Einzelnen den Rahmen dieses Beitrags sprengen würde. Die weiter unten präsentierten Ergebnisse basieren auf einer Variante des „nearestneighour-matching" ohne Zurücklegen, das heißt, für jeden Betrieb mit Betriebsrat wird ein Nachbar aus den Betrieben ohne Betriebsrat gesucht, der diesem am ähnlichsten ist. Ohne Zurücklegen bedeutet, dass ein bestimmter Betrieb ohne Betriebsrat nur einmal der nächste Nachbar sein kann. Alternativ wurden auch andere Matching-Varianten mit Zurücklegen berechnet, um die Sensitivität der Ergebnisse abschätzen zu können. In Tabelle 2 ist die Robustheit der einzelnen Resultate dokumentiert; sie wird in der Interpretation berücksichtigt.

Das konkrete Verfahren besteht im Wesentlichen aus zwei Schritten. In einem ersten vorbereitenden Schritt wird die Wahrscheinlichkeit für die Existenz eines Betriebsrats durch Probit-Schätzungen auf Basis der beobachteten und als relevant erachteten Merkmale bestimmt. Die eigentliche Paarbildung erfolgt dann mit den geschätzten Koeffizienten als Maß der Distanz zwischen den Betrieben. ${ }^{4}$

2 Für eine ausführliche Darstellung des IAB-Betriebspanels siehe Bellmann 2002.

3 Für einen Einstieg in die methodische Diskussion zu den Matching-Verfahren vgl. z. B. Rosenbaum/ Rubin 1985, Heckman/Lalonde/Smith 1999, Smith/Todd 2000.

$4 \quad$ Als unabhängige Variable für die Probitschätzungen fanden die in der Literatur üblicherweise als mögliche Bestimmungsfaktoren herangezogenen Merkmale Eingang (Frick/Sadowski 1995; Addison et al. 2002; Bellmann/Ellguth 2006). Das sind neben betriebsstrukturellen Merkmalen - wie der Betriebsgröße, dem Filialstatus, der Rechtsform, einer möglichen Mitgliedschaft in der Handwerkskammer, dem technischen Stand der Anlagen, der regionalen Lage und dem Alter des Betriebs - vor allem Variablen der Beschäftigtenstruktur. Darüber hinaus wird eine mögliche Tarifbindung und natürlich die Branchenzugehörigkeit berücksichtigt, ebenso die Information, ob der Betrieb sich in ausländischem (für Ostdeutschland auch, ob er sich in ostdeutschem) Eigentum befindet. 
Tabelle 1: Mittelwertvergleiche der Betriebe mit Betriebsrat und solchen ohne vor und nach dem Matching1

\begin{tabular}{|c|c|c|c|c|c|c|c|c|c|c|}
\hline & \multicolumn{5}{|c|}{ Westdeutschland ${ }^{2}$} & \multicolumn{5}{|c|}{ Ostdeutschland 3} \\
\hline & $\begin{array}{c}\text { Alle } \\
\text { Betriebe } \\
\text { mit } B R\end{array}$ & $\begin{array}{c}\text { Alle } \\
\text { Betriebe } \\
\text { ohne BR }\end{array}$ & $\begin{array}{c}\text { Zwillings- } \\
\text { Betriebe } \\
\text { mit BR }\end{array}$ & $\begin{array}{l}\text { Zwillings- } \\
\text { Betriebe } \\
\text { ohne BR }\end{array}$ & $\begin{array}{c}|t| \\
\operatorname{diff} \sim=0\end{array}$ & $\begin{array}{c}\text { Alle } \\
\text { Betriebe } \\
\text { mit BR }\end{array}$ & $\begin{array}{c}\text { Alle } \\
\text { Betriebe } \\
\text { ohne BR }\end{array}$ & $\begin{array}{l}\text { Zwillings- } \\
\text { Betriebe } \\
\text { mit BR }\end{array}$ & $\begin{array}{l}\text { Zwillings- } \\
\text { Betriebe } \\
\text { ohne BR }\end{array}$ & $\begin{array}{c}|t| \\
\operatorname{diff} \sim=0\end{array}$ \\
\hline Betriebsgröße & 156 & 37 & 87 & 83 & 1,25 & 137 & 36 & 80 & 86 & 1,30 \\
\hline Filialbetrieb/Mittelinstanz & 0,42 & 0,11 & 0,30 & 0,28 & 1,21 & 0,44 & 0,11 & 0,34 & 0,29 & 1,74 \\
\hline Einzeluntern./Personengesellschaft & 0,01 & 0,36 & 0,05 & 0,06 & 1,58 & 0,01 & 0,34 & 0,01 & 0,01 & 0,24 \\
\hline Handwerksbetrieb & 0,10 & 0,26 & 0,15 & 0,16 & 1,02 & 0,06 & 0,34 & 0,09 & 0,10 & 0,56 \\
\hline Gründung vor 90 & 0,82 & 0,66 & 0,77 & 0,75 & 1,33 & 0,34 & 0,20 & 0,22 & 0,19 & 1,32 \\
\hline Gründung nach 02 & 0,08 & 0,06 & 0,08 & 0,07 & 0,03 & 0,05 & 0,09 & 0,07 & 0,07 & 0,32 \\
\hline Frauenanteil & 0,40 & 0,45 & 0,42 & 0,42 & 0,42 & 0,49 & 0,38 & 0,46 & 0,42 & 0,79 \\
\hline Anteil Qualifizierter & 0,75 & 0,63 & 0,71 & 0,70 & 1,44 & 0,86 & 0,74 & 0,82 & 0,79 & 1,16 \\
\hline Technischer Stand (gut/sehr gut = 1) & 0,68 & 0,68 & 0,69 & 0,68 & 0,42 & 0,73 & 0,69 & 0,74 & 0,71 & 1,37 \\
\hline Regionale Lage (Kern > 50.000) & 0,53 & 0,48 & 0,49 & 0,50 & 0,47 & 0,37 & 0,29 & 0,32 & 0,35 & 0,88 \\
\hline Tarifbindung & 0,82 & 0,45 & 0,68 & 0,65 & 1,64 & 0,78 & 0,31 & 0,60 & 0,57 & 1,12 \\
\hline In ausländischem Eigentum & 0,10 & 0,04 & 0,06 & 0,07 & 1,36 & 0,09 & 0,03 & 0,06 & 0,07 & 0,22 \\
\hline \multirow[t]{2}{*}{ In ostdeutschem Eigentum } & & & & & & 0,29 & 0,74 & 0,42 & 0,44 & 0,78 \\
\hline & 2574 & 4203 & 1313 & 1313 & & 1264 & 2609 & 648 & 648 & \\
\hline $\begin{array}{l}\text { **** signalisieren einen signifikanten Unterschied auf } \\
1 \text { privatwirtschaftliche Betriebe mit } 5 \text { bis } 500 \text { Beschäftig } \\
2 \text { In Westdeutschland wurden aus den beiden Gruppen } \\
3 \text { In Ostdeutschland wurden aus den beiden Gruppen v } \\
\text { Quelle: IAB-Betriebspanel } 2006 \text {. }\end{array}$ & $\begin{array}{l}\text { m } 1 \%-/ 5 \% \\
\text { en ohne Lan } \\
\text { /on Betriebe } \\
\text { n Betrieben }\end{array}$ & $\begin{array}{l}\text { veau. } \\
\text { irtschaft un } \\
\text { it (2574) u } \\
\text { t(1264) un }\end{array}$ & $\begin{array}{l}\text { Org. o. Erwe } \\
\text { ohne }(4203 \\
\text { ohne (2609) }\end{array}$ & $\begin{array}{l}\text { zweck. } \\
\text { etriebsrat } 13 \\
\text { triebsrat } 648\end{array}$ & $\begin{array}{l}3 \text { Paare ge } \\
\text { Paare gebilc }\end{array}$ & $\begin{array}{l}\text { ziehung oh } \\
\text { lung ohne }\end{array}$ & $\begin{array}{l}\text { Zurücklegen } \\
\text { rücklegen). }\end{array}$ & & | & $\begin{array}{l}\text { Os Böckler } \\
\text { ftung }\end{array}$ \\
\hline
\end{tabular}

Allerdings gilt für das hier verwendete Verfahren eine Einschränkung, die der Struktur der Betriebe ohne Betriebsrat geschuldet ist. Der Umstand, dass es ab einer bestimmten Betriebsgröße kaum noch Betriebe gibt, die über keine Interessenvertretung verfügen, schränkt das Reservoir an Kandidaten, die für die Paarbildung zur Verfügung stehen, so stark ein, dass eine erfolgreiche Paarbildung nicht zu bewerkstelligen ist, wenn alle Betriebe (mit Betriebsrat) einbezogen würden. Daher wurden nur Betriebe bis zu einer Größe von 500 Beschäftigten berücksichtigt. ${ }^{5}$

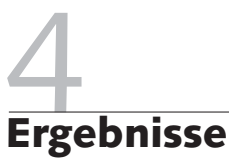

Aus Tabelle 1 ist ersichtlich, dass das Matching-Verfahren als erfolgreich bezeichnet werden kann, da eine gute Übereinstimmung der beiden Gruppen in Bezug auf die relevanten Variablen vorliegt, die sich vorher zum Teil dramatisch unterschieden haben. ${ }^{6}$ Selbst in Bezug auf die Betriebsgröße und andere strukturelle Merkmale verbleiben keine interpretierbaren Unterschiede mehr. Mögliche Differenzen in den interessierenden „Performance-Merkmalen“können nun mit der Existenz eines Betriebsrats in Verbindung gebracht werden. Dabei wird - anders als sonst üblich - kein Anspruch auf die Identifizierung kausaler Effekte erhoben. Der Grund hierfür ist, dass die kausale Interpretation solcher Unter- schiede als Ausgangspunkt eines sogenannten Treatments bedarf. In unserem Zusammenhang wäre das die Neugründung von Betriebsräten. Da es davon aber zu wenige Fälle gibt, können wir nur die Existenz eines Betriebsrats betrachten und entsprechende Zwillingsbetriebe identifizieren. In der Konsequenz beschränken wir uns auch auf eine Interpretation, die die auftretenden Differenzen im Sinne eines stark verfeinerten Kontrollgruppenansatzes vor dem Hintergrund bereits vorliegender quantitativer und qualitativer Erkenntnisse deutet.

Ein Vergleich der betrieblichen Arbeitszeitvariablen zeigt, dass zumindest bei einigen signifikante Differenzen zwischen den Betrieben mit und „Zwillingsbetrieben“ ohne Betriebsrat bestehen bleiben (Tabelle 2). Die Ergebnisse lassen denn auch einige interessante Rückschlüsse auf das Wirken der betrieblichen Interessenvertretung $\mathrm{zu}$, vor allem wenn sie in den weiteren Rahmen betrieblicher Flexibilisierungsbemühungen eingebettet werden.

Wie beeinflusst nun der Betriebsrat die betriebliche Arbeitszeitsituation? Hinsichtlich der Wochenarbeitszeiten ist für Westdeutschland festzustellen, dass das Vorhandensein des Betriebsrats bei sonst gleichen Charakteristika des Betriebs die Länge der Wochenarbeitszeit signifikant verringert und zwar um durchschnittlich 0,6 Stunden pro Woche. Für Ostdeutschland weisen die Ergebnisse in die gleiche Richtung, sind jedoch nicht robust in den verschiedenen alternativen Matchingverfahren. Betriebsräte wirken also zusätzlich zur tariflichen Arbeitszeitvorgabe senkend auf die Wochenarbeitszeiten. Plausibel und auf Basis von Theorie und Fallstudienevidenz gut nachvollziehbar ist die Erklärung, dass Betriebsräte - konform mit dem Betriebsverfassungsgesetz - im Betrieb als Kontrollinstanzen der tariflichen Bestimmungen fungieren; erst ihr Eingreifen sorgt dafür, dass die tariflichen Normen faktisch wirksam werden. Ein konkreterer Erklärungsansatz wäre, dass Betriebsräte im Rahmen von Korridormodellen und betrieblichen Bündnissen für Arbeit auch an temporären Unterschreitungen der tariflichen Arbeitszeitniveaus beteiligt sind. Hierfür spricht auch die gegenüber der betriebsratsfreien Vergleichsgruppe stark erhöhte Nutzung von Arbeitszeitkonten in den Betrieben mit Betriebsrat.

Dieser Befund gilt im Westen wie im Osten Deutschlands und stimmt auch mit bisherigen Ergebnissen überein (Ludewig 2001). Hinsichtlich der Inklusivität der Konten sind auf Basis unserer Daten keine

\footnotetext{
Die bereits erwähnten Sensitivitätstests umfassten auch Matching-Varianten für ein eingeschränktes Größensegment der Betriebe mit 21-199 Beschäftigten.

6 Zur Übersichtlichkeit ist die Darstellung auf solche Variablen beschränkt, die in beiden Landesteilen einen signifikanten Erklärungsbeitrag liefern (Ausnahme: Betrieb in ostdeutschem Eigentum, für die es in Westdeutschland zu wenige Beobachtungen gibt). Aber auch alle übrigen Variablen zeigen nach der Paarbildung keine signifikanten Unterschiede.
} 
Tabelle 2: Mittelwertvergleiche zum Arbeitszeitreglement der "Zwillingsbetriebe ${ }^{\text {A }}$ mit und ohne Betriebsrat1

\begin{tabular}{|c|c|c|c|c|c|c|c|c|c|c|c|c|}
\hline & \multicolumn{6}{|c|}{ Westdeutschland ${ }^{2}$} & \multicolumn{6}{|c|}{ Ostdeutschland ${ }^{3}$} \\
\hline & $\begin{array}{c}\text { Alle } \\
\text { Betriebe } \\
\text { mit } B R\end{array}$ & $\begin{array}{c}\text { Alle } \\
\text { Betriebe } \\
\text { ohne BR }\end{array}$ & $\begin{array}{c}\text { Zwillings- } \\
\text { Betriebe } \\
\text { mit BR }\end{array}$ & $\begin{array}{l}\text { Zwillings- } \\
\text { Betriebe } \\
\text { ohne BR }\end{array}$ & $\begin{array}{c}|t| \\
\operatorname{diff} \sim=0\end{array}$ & $\begin{array}{l}\text { Ergebnis } \\
\text { robust? }\end{array}$ & $\begin{array}{c}\text { Alle } \\
\text { Betriebe } \\
\text { mit BR }\end{array}$ & $\begin{array}{c}\text { Alle } \\
\text { Betriebe } \\
\text { ohne } B R\end{array}$ & $\begin{array}{c}\text { Zwillings- } \\
\text { Betriebe } \\
\text { mit BR }\end{array}$ & $\begin{array}{c}\text { Zwillings- } \\
\text { Betriebe } \\
\text { ohne BR }\end{array}$ & $\begin{array}{c}|t| \\
\operatorname{diff} \sim=0\end{array}$ & $\begin{array}{l}\text { Ergebnis } \\
\text { robust? }\end{array}$ \\
\hline Vereinbarte Wochen-AZ & 38,3 & 39,4 & 38,6 & 39,2 & $7,73^{* *}$ & $\mathrm{Ja}$ & 39,3 & 39,9 & 39,3 & 39,7 & $5,26^{* *}$ & Nein \\
\hline AZ-Konten & 0,71 & 0,32 & 0,64 & 0,46 & $9,29 * *$ & Ja & 0,68 & 0,38 & 0,62 & 0,51 & $3,83^{* *}$ & Ja \\
\hline Nutzungsintensität der AZK & 0,83 & 0,83 & 0,81 & 0,82 & 0,34 & $\mathrm{Ja}$ & 0,82 & 0,82 & 0,84 & 0,81 & 1,94 & $\mathrm{Ja}$ \\
\hline Bezahlte Überstunden & 0,54 & 0,31 & 0,49 & 0,48 & 0,47 & $\mathrm{Ja}$ & 0,39 & 0,33 & 0,39 & 0,44 & 1,64 & Ja \\
\hline Nutzungsintensität bez. ÜS & 0,31 & 0,44 & 0,33 & 0,42 & $5,04 * *$ & $\mathrm{Ja}$ & 0,33 & 0,53 & 0,38 & 0,47 & $3,02 * *$ & Nein \\
\hline In Freizeit abgegoltene ÜS & 0,76 & 0,50 & 0,72 & 0,65 & $4,18^{* *}$ & $\mathrm{Ja}$ & 0,63 & 0,54 & 0,63 & 0,59 & 1,43 & Nein \\
\hline Teilzeit & 0,93 & 0,87 & 0,91 & 0,91 & 0,82 & $\mathrm{Ja}$ & 0,85 & 0,70 & 0,80 & 0,77 & 1,15 & Nein \\
\hline Nutzungsintensität Teilzeit & 0,23 & 0,32 & 0,26 & 0,25 & 1,29 & $\mathrm{Ja}$ & 0,26 & 0,26 & 0,26 & 0,25 & 0,76 & Nein \\
\hline Sozpflichtige TZ & 0,82 & 0,54 & 0,75 & 0,63 & $6,96 * *$ & $\mathrm{Ja}$ & 0,74 & 0,44 & 0,67 & 0,54 & $4,72 * *$ & $\mathrm{Ja}$ \\
\hline Nutzungsintensität sozpfl. TZ & 0,21 & 0,23 & 0,25 & 0,21 & $3,60 * *$ & Nein & 0,26 & 0,26 & 0,28 & 0,26 & 1,24 & Nein \\
\hline Geringfügige TZ & 0,52 & 0,69 & 0,55 & 0,70 & $8,07^{* *}$ & $\mathrm{Ja}$ & 0,40 & 0,43 & 0,38 & 0,46 & $3,28^{* *}$ & $\mathrm{Ja}$ \\
\hline Nutzungsintensität geringf. TZ & 0,09 & 0,24 & 0,10 & 0,15 & $5,68^{* *}$ & $\mathrm{Ja}$ & 0,07 & 0,17 & 0,08 & 0,12 & $3,88^{* *}$ & Nein \\
\hline Vertrauens-AZ & 0,27 & 0,22 & 0,25 & 0,24 & 0,45 & Ja & 0,19 & 0,11 & 0,17 & 0,14 & 1,45 & Ja \\
\hline Nutzungsintensität VAZ & 0,39 & 0,70 & 0,44 & 0,53 & $2,80^{* *}$ & Nein & 0,45 & 0,66 & 0,53 & 0,54 & 0,11 & $\mathrm{Ja}$ \\
\hline Samstagsarbeit & 0,35 & 0,35 & 0,34 & 0,36 & 0,58 & Nein & 0,32 & 0,30 & 0,28 & 0,28 & 0,00 & Ja \\
\hline Sonntagsarbeit & 0,23 & 0,17 & 0,22 & 0,20 & 0,92 & Nein & 0,25 & 0,15 & 0,21 & 0,18 & 1,33 & $\mathrm{Ja}$ \\
\hline \multirow[t]{2}{*}{ Beschäftigungssichernde AZV } & 0,06 & 0,02 & 0,06 & 0,02 & $4,42 * *$ & $\mathrm{Ja}$ & 0,14 & 0,04 & 0,09 & 0,05 & $2,68^{* *}$ & Nein \\
\hline & V 2574 & 4203 & 1313 & 1313 & & & 1264 & 2609 & 648 & 648 & & \\
\hline \multicolumn{13}{|c|}{$\begin{array}{l}\text { **/* signalisieren einen signifikanten Unterschied auf dem } 1 \%-/ 5 \% \text {-Niveau } \\
1 \text { privatwirtschaftliche Betriebe mit } 5 \text { bis } 500 \text { Beschäftigte ohne Landwirtschaft und Org. O. Erwerbszweck. } \\
2 \text { In Westdeutschland wurden aus den beiden Gruppen von Betrieben mit (2574) und ohne (4203) Betriebsrat } 1313 \text { Paare gebildet (Ziehung ohne Zurücklegen). } \\
3 \text { In Ostdeutschland wurden aus den beiden Gruppen von Betrieben mit (1264) und ohne (2609) Betriebsrat } 648 \text { Paare gebildet (Ziehung ohne Zurücklegen). } \\
\text { Quelle: IAB-Betriebspanel 2006. }\end{array}$} \\
\hline
\end{tabular}

signifikanten Unterschiede feststellbar. Thesen, nach denen Betriebsräte für eine breitere Einbeziehung der Mitarbeiter in Kontenregelungen sorgen, bestätigen sich also nicht - oder nicht mehr. Denn der Grad der Inklusivität von Arbeitszeitkonten - gemessen am Anteil der einbezogenen Beschäftigten - nähert sich bereits der Obergrenze des betrieblich Möglichen wie die deskriptiven Auswertungen weiter vorne zeigen. Fallstudien wie repräsentative Untersuchungen aus den vergangenen Jahren belegen, dass mittlerweile auch solche betrieblichen Bereiche flexible Arbeitszeitregelungen haben, in denen die Einführung von Konten und Gleitzeit noch vor 15 Jahren als unmöglich galt, so etwa Fertigungsbereiche im Verarbeitenden Gewerbe (Promberger et al. 2002). Einschränkend ist hierzu anzumerken, dass der Zeitkontenbegriff im IAB-Betriebspanel auch reine Überstundenkonten umfasst. Eine Rolle des Betriebsrats als Motor neuer Formen der Arbeitszeitflexibilisierung ist deshalb nicht direkt identifizierbar. Was sich im Zusammenhang mit Betriebsräten allerdings zeigt, ist ein vermehrtes Auftreten formalisierter Arbeitszeitregelungen, deren Gültigkeit damit auch von den Beschäftigten eingefordert, ja arbeitsgerichtlich eingeklagt werden kann. Durch die - wie auch immer geartete - Einbeziehung der Wünsche von Beschäftigten „ex ante“ kann zudem eine für betriebliche Flexibilisierung und Stabi- lisierung unerlässliche - Reduzierung möglicher Konfliktpotenziale und -kosten durch Betriebsräte erfolgen.

Hinsichtlich der Verbreitung von Teilzeitarbeit generell sind die Befunde nicht schlagend; westdeutsche Betriebe mit Betriebsräten praktizieren jedoch häufiger sozialversicherungspflichtige Teilzeit und deutlich seltener geringfügige Teilzeitarbeit. Gleichermaßen dämpft der Betriebsrat die Anteile geringfügig Beschäftigter. Betriebsräte fördern also „gute " und hemmen „prekäre“ Teilzeitbeschäftigung (Düll/ Ellguth 1999). ${ }^{7}$

In unserer vorangegangenen Studie hatte sich gezeigt (Ellguth/Promberger 2004b), dass Betriebsräte in Ostdeutschland vor allem in Richtung eines Ausbaus von Vollzeitbeschäftigung optieren; dahinter wurde ein Zusammenhang mit arbeitskulturellen Besonderheiten (Präferenz von Vollzeitarbeit auch bei Frauen) und mit dem generell niedrigeren Entgeltniveau in Ostdeutschland vermutet. Unterstützt wurde diese Interpretation durch die Tatsache, dass zwar genauso viele Betriebe mit Betriebsrat Teilzeitarbeit praktizierten wie Betriebe ohne Betriebsrat, die Nutzungsintensität (der „guten“ Teilzeitarbeit) jedoch in Betriebsratsbetrieben deutlich niedriger ausfiel. Diese Beziehung kann in der vorliegenden Analyse nicht mehr identifiziert werden. Es zeigt sich kein Zusammenhang mehr zwischen dem Vorhanden- sein von Betriebsräten und der Nutzungsintensität ,guter“ Teilzeitarbeit in ostdeutschen Betrieben. Nach wie vor fördert also der Betriebsrat die Teilzeitwünsche der Beschäftigten nach "guter“ Teilzeit in Westdeutschland. Betriebsratsbetriebe in Ostdeutschland gleichen sich diesem Muster offenbar mehr und mehr an.

In West- wie Ostdeutschland lässt sich kein Einfluss des Betriebsrats auf die grundsätzliche Entscheidung, bezahlte Überstunden einzusetzen, feststellen. Hingegen ist die Intensität der Nutzung bezahlter Überstunden, gemessen am Anteil der betroffenen Beschäftigten, in Betrieben mit Betriebsrat geringer als in solchen ohne (Westdeutschland). Ob diese dämpfende Wirkung der Interessenvertretung eher auf die Berücksichtigung von Belegschaftswünschen zurückzuführen oder aber Ausdruck betriebspolitischer Erwägungen ist, lässt sich an dieser Stelle nicht sagen. Vermutungen, dass aufgrund der Einkommenssituation in Ostdeutschland die Beschäftigten häufiger als in Westdeutschland bezahlte Überstunden machen wollen und

\footnotetext{
Dies ließe sich aber auch dahingehend interpretieren, dass geringfügige Beschäftigung eben vorwiegend in einem Segment der Betriebslandschaft eingesetzt wird, in dem es zumeist auch keinen Betriebsrat gibt. Der vorliegende Zusammenhang wäre also weniger dem aktiven Eingreifen der Interessenvertretungen geschuldet.
} 
dies auch über ihre Interessenvertretung durchsetzen, können auf Basis unserer Ergebnisse nicht bestätigt werden (Schank/ Schnabel 2004).

Der Umstand, dass der Einsatz von in Freizeit ausgeglichenen Überstunden eher in Betrieben mit Betriebsrat geschieht (wiederum mit einem für Ostdeutschland nicht robusten Ergebnis), könnte mit der vermeintlichen Schutzfunktion des Betriebsrats erklärt werden. Dieser sorgt unter Umständen für ein häufigeres „Abfeiern"von Überstunden, nicht zuletzt aus gesundheitlichen Aspekten.

Vertrauensarbeitszeit ist eine Arbeitszeitform, die von Betriebsräten meist eher skeptisch beurteilt wird (Böhm et al. 2004), sodass ein verringertes Auftreten in Betriebsratsbetrieben vermutet werden kann. Es lässt sich jedoch kein derartiger Unterschied finden. Eine plausible Schlussfolgerung hierfür wäre, dass Betriebsräte diese vor allem bei „privilegierten“ Beschäftigtengruppen eingesetzte Arbeitszeitregulierung nicht beeinflussen wollen oder können.

Auch hinsichtlich Samstags- und Sonntagsarbeit lassen sich interessanterweise kaum bzw. keine robusten Unterschiede zwischen Betrieben mit und ohne Betriebsrat feststellen. Offensichtlich gehorcht der Einsatz dieser „ungünstigen“ Arbeitszeitformen Imperativen, die im etablierten System der industriellen Beziehungen auf der Betriebsebene nicht oder nicht wirksam verhandelt werden. Eine Betrachtung der Nutzungsintensitäten könnte möglicherweise zu anderen Ergebnissen kommen, doch hierzu liegen in den vorliegenden Daten keine Angaben vor.

Last but not least bestätigen sich die Vermutungen, dass beschäftigungssichernde Arbeitszeitverkürzungen, die in der Regel über tarifliche Öffnungsklauseln verhandelt werden, an die Existenz eines Betriebsrats gekoppelt sind - auch wenn sich die Verbreitung dieser Vereinbarungen insgesamt auf niedrigem Niveau bewegt (Seifert/Massa-Wirth 2005).

Insgesamt ergibt unsere MatchingAnalyse - von der Überstundenfrage abgesehen - ein durchaus konsistentes Bild der Auswirkungen der Existenz eines Betriebsrats auf die Arbeitszeitsituation im Betrieb: Die Einflüsse des Betriebsrats sind unverkennbar und nicht auf andere Variablen zurückzuführen. Sie erhalten ihr charakteristisches Gepräge jedoch von den regionalen Betriebskulturen und Arbeitsmärkten. In der ganzen Bundesrepublik fördern Betriebsräte die Existenz von Zeitkonten. Sie stabilisieren "gute“, sozialversicherungspflichtige Teilzeitarbeit und begrenzen entsprechend das Ausmaß geringfügiger Beschäftigung. Dabei stehen Betriebsräte den betrieblichen Anpassungs- und Flexibilisierungsbestrebungen anscheinend nicht im Wege; dies sieht man unter anderem an der Verbreitung von Vertrauensarbeitszeitregelungen und Wochenendarbeit.

Abschließend drängt sich die Frage auf, ob sich die ermöglichende, sozial integrative und mäßigende bzw. dämpfende Funktion von Betriebsräten, die wir für die Arbeitszeitpolitik festgestellt haben, vielleicht auch auf andere Sphären der betrieblichen Arbeitspolitik übertragen lässt. Sollte dies der Fall sein, würde die Existenz oder Nichtexistenz von Betriebsräten einen entscheidenden Indikator für die Qualität von Arbeitsplätzen darstellen. Dies zu klären, betrachten wir als eine unserer nächsten Aufgaben.

\section{LITERATUR}

Addison, J. T./Bellmann, L./Schnabel, C./Wagner, J. (2003): German Works Councils Old and New: Incidence, Coverage and Determinants. Schmollers Jahrbuch 123, S. 339-358

Artus, I. (2003): Die Kooperation zwischen Betriebsräten und Gewerkschaften als neuralgischer Punkt des Tarifsystems. Eine exemplarische Analyse am Beispiel Ostdeutschlands, in: Industrielle Beziehungen 10, S. 250-272

Artus, I./Liebold, R./Lohr, K./Schmidt, E./Schmidt, R./Strohwald, U. (2001): Betriebliches Interessenhandeln, Zur politischen Kultur der Austauschbeziehungen zwischen Management und Betriebsrat in der ostdeutschen Industrie Bd. 2, Opladen

Bellmann, L. (2002): Das IAB-Betriebspanel - Konzeption und Anwendungsbereiche, in: Allgemeines Statistisches Archiv 86, S. 177-188 Bellmann, L./Ellguth, P. (2006): Verbreitung von Betriebsräten und ihr Einfluss auf die betriebliche Weiterbildung, in: Jahrbücher für die Nationalökonomie und Statistik 5, S. 487-504

Bergmann, J./Jacobi, O./Müller-Jentsch, W. (1975): Gewerkschaften in der Bundesrepublik. Gewerkschaftliche Lohnpolitik zwischen Mitgliederinteressen und Systemzwängen, Frankfurt
Böhm, S./Herrmann, C./Trinczek, R. (2004): Herausforderung Vertrauensarbeitszeit. Zur Kultur und Praxis eines neuen Arbeitszeitmodells, Berlin

Blume, O. (1964): Normen und Wirklichkeit einer Betriebsverfassung, Tübingen

Bosch, G./Engelhardt, N./Hermann, K./Kurz-Scherf, I./Seifert, H. (1988): Arbeitszeitverkürzung im Betrieb, Köln

Bosch, A./Ellguth, P./Schmidt, R./Trinczek, R. (1999): Betriebliches Interessenhandeln. Zur politischen Kultur der Austauschbeziehungen zwischen Management und Betriebsrat in der westdeutschen Industrie, Opladen

Düll, H./Ellguth, P. (1999): Betriebliche Strukturen der Teilzeitbeschäftigung in West- und Ostdeutschland, in: Mitteilungen aus der Arbeitsmarkt- und Berufsforschung 3, S. 269-280

Ellguth, P. (2007): Betriebliche und überbetriebliche Interessenvertretung: Ergebnisse aus dem IAB-Betriebspanel 2005, in: WSI-Mitteilungen 3, S. 155-157 
Ellguth, P./Promberger, M. (2004a): Arbeitszeiten in der Dienstleistungswirtschaft, in: Mönig-Raane, M./Sterkel, G./Wiedemuth, J. (Hrsg.): Es ist Zeit. Logbuch für die ver.di-Arbeitszeitinitiative, Hamburg

Ellguth, P./Promberger, M. (2004b): Arbeitszeitsituation und Betriebsrat eine Matched-Pairs-Analyse mit Daten des IAB-Betriebspanels, in: Bellmann, L./Schnabel, C. (Hrsg.): Betriebliche Arbeitszeitpolitik im Wandel. Beiträge zur Arbeitsmarkt- und Berufsforschung 288, Nürnberg, S. 111131

Frege, C. M. (2002): A Critical Assessment of the Theoretical and Empirical Research on German Works Councils, in: British Journal of Industrial Relations 40, pp. 221-248

Freeman, R. B./Lazear, E. P. (1995): An Economic Analysis of Works Councils, in: Rogers, J./Streek, W. (eds.): Works Councils - Consultation, Representation and Operation in Industrial Relations, Chicago, pp. 27-52 Frick, B./Sadowski, D. (1995): Works Councils, Unions, and Firm Performance: The Impact of Workers' Participation in Germany, in: Buttler, F./Franz, W./Schettkat, R./Soskice, D. (eds.): Institutional Frameworks and Labor Market Performance: Comparative Views on the U. S. and German Economies, London, pp. 46-81

Fürstenberg, F. (1958): Der Betriebsrat - Strukturanalyse einer Grenzinstitution, in: Kölner Zeitschrift für Soziologie und Sozialpsychologie, S. 418-429

Heckmann, J. J./Lalonde, R. J./Smith, J. A. (1999): The Economics and Econometrics of Aktive Labor Market Programms, in: Ashenfelder O./Card, D. (eds.): Handbook of Labor Economics, Amsterdam, pp. 1865-2097

Herrmann, C./Promberger, M./Singer, S./Trinczek, R. (1999): Forcierte Arbeitszeitflexibilisierung, Berlin

Hübler, O. (2003): Fördern oder behindern Betriebsräte die Unternehmensentwicklung?, in: Perspektiven der Wirtschaftspolitik 4, S. 379-397 Jirjahn, U. (2005): Ökonomische Wirkungen des novellierten Betriebsverfassungsgesetzes - Was können wir vor dem Hintergrund zunehmender Globalisierung und veränderter arbeitsorganisatorischer Bedingungen erwarten?, in: Zeitschrift für Arbeitsmarktforschung 2/3, S. 241-267 Kotthoff, H. (1979): Zum Verhältnis von Betriebsrat und Gewerkschaft. Ergebnisse einer empirischen Untersuchung, in: Bergmann, J. (Hrsg.): Beiträge zur Soziologie der Gewerkschaften, Frankfurt Kotthoff, H. (1981): Betriebsräte und betriebliche Herrschaft. Eine Typologie von Partizipationsmustern im Industriebetrieb, Frankfurt Kotthoff, H. (1994): Betriebsräte und Bürgerstatus. Wandel und Kontinuität betrieblicher Mitbestimmung, München und Mering Lehndorff, S. (1997): Zeitnot und Zeitsouveränität in der Just-in-TimeFabrik, München und Mering

Lindecke, C. (2000): Flexible Arbeitszeitorganisation in der Praxis. Eine Untersuchung in sechs Unternehmen, München und Mering Ludewig, O. (2001): Betriebliche Bestimmungsgründe von Arbeitszeitkonten, in: Mitteilungen aus der Arbeitsmarkt- und Berufsforschung 3, S. 302-313
Matthies, H./Mückenberger, U./Offe, C./Peter, E./Raasch, S. (1994): Arbeit 2000. Anforderungen an eine Neugestaltung der Arbeitswelt, Reinbeck

Mausolff, A. (1952): Gewerkschaft und Betriebsrat im Urteil der Arbeitnehmer, Darmstadt

Müller-Jentsch, W. (1995): From Collective Voice to Co-Manager, in: Rogers, J./Streeck, W. (eds.): Works Councils: Consultation, Representation and Cooperation in Industrial Relations. Chicago, London, pp. 53-78 Müller-Jentsch, W./Seitz, B. (1998): Betriebsräte gewinnen Konturen. Ergebnisse einer Betriebsrätebefragung, in: Industrielle Beziehungen, 4 , S. 361-378

Neuloh, O. (1956): Die deutsche Betriebsverfassung und ihre Sozialformen bis zur Mitbestimmung, Tübingen

Pirker, T./Braun, S./Lutz, B./Hammelrath, F. (1955): Arbeiter-Management-Mitbestimmung, Stuttgart

Popitz, H./Bahrdt, H.-P./Jüres, E.-A./Kesting, H. (1964): Technik und Industriearbeit. Soziologische Untersuchungen in der Hüttenindustrie, Tübingen

Promberger, M. (1993): Was wird aus der Arbeitszeit?, München und Mering

Promberger, M. (2002): Das VW-Modell und seine Nachfolger - Pioniere einer neuartigen Beschäftigungspolitik, München und Mering

Promberger, M./Böhm, S./Heyder, T./Pamer, S./Strauß, K. (2002): Hochflexible Arbeitszeiten in der Industrie - Chancen, Risiken und Grenzen für Beschäftigte, Berlin

Promberger, M. (2004): Wie neuartig sind flexible Arbeitszeiten? - Historische Grundlinien der Arbeitszeitpolitik, in: Seifert, H. (Hrsg.): Arbeitszeitflexibilisierung, Frankfurt/M.

Rosenbaum, P. R./Rubin, D. B. (1985): Constructing a Control Group Using Multivariate Matched Sampling Methods that Incorporate the Propensity Score, The American Statistican, pp. 33-38

Schank, T./Schnabel, C. (2004): Betriebliche Determinanten des Überstundeneinsatzes, in: Beiträge zur Arbeitsmarkt- und Berufsforschung 288, Nürnberg, S. 37-62

Schmidt, R./Trinczek, R. (1988): Verbetrieblichung - viele Risiken, wenig Chancen. Erfahrungen aus der Umsetzung der 38,5-Stunden-Woche, in: Hildebrandt, E./Sperling, J. (Hrsg.): Zweidrittelgesellschaft - Eindrittelgewerkschaft. Kritisches Gewerkschaftsjahrbuch 1988/89, Berlin, S. 5472

Schmidt, R./Trinczek, R. (1991): Duales System: Tarifliche und betriebliche Interessenvertretung, in: Müller-Jentsch, W. (Hrsg.): Konfliktpartnerschaft. Akteure und Institutionen der industriellen Beziehungen, München und Mering, S. 167-199

Seifert, H./Massa- Wirth, H. (2005): Pacts for Employment and Competitiveness in Germany, in: Industrial Relations Journal, S. 217-240. Smith, J. /Todd, P. (2000): Does Matching Overcome LaLonde's Critique of Nonexperimental Estimators, Working Paper 